\title{
Steiner Forest Orientation Problems *
}

\author{
Marek Cygan ${ }^{1 \star \star}$, Guy Kortsarz ${ }^{2 \star \star \star}$, and Zeev Nutov ${ }^{3}$ \\ 1 IDSIA, University of Lugano, Switzerland marek@idsia.ch \\ ${ }^{2}$ Rutgers University, Camden guyk@camden.rutgers.edu \\ 3 The Open University of Israel nutov@openu.ac.il
}

\begin{abstract}
We consider connectivity problems with orientation constraints. Given a directed graph $D$ and a collection of ordered node pairs $P$ let $P[D]=\{(u, v) \in P: D$ contains a $u v$-path $\}$. In the Steiner Forest Orientation problem we are given an undirected graph $G=(V, E)$ with edge-costs and a set $P \subseteq V \times V$ of ordered node pairs. The goal is to find a minimum-cost subgraph $H$ of $G$ and an orientation $D$ of $H$ such that $P[D]=P$. We give a 4-approximation algorithm for this problem. In the Maximum Pairs Orientation problem we are given a graph $G$ and a multi-collection of ordered node pairs $P$ on $V$. The goal is to find an orientation $D$ of $G$ such that $|P[D]|$ is maximum. Generalizing the result of Arkin and Hassin [DAM'02] for $|P|=2$, we will show that for a mixed graph $G$ (that may have both directed and undirected edges), one can decide in $n^{O(|P|)}$ time whether $G$ has an orientation $D$ with $P[D]=P$ (for undirected graphs this problem admits a polynomial time algorithm for any $P$, but it is NP-complete on mixed graphs). For undirected graphs, we will show that one can decide whether $G$ admits an orientation $D$ with $|P[D]| \geq k$ in $O(n+m)+2^{O(k \cdot \log \log k)}$ time; hence this decision problem is fixed-parameter tractable, which answers an open question from Dorn et al. [AMB'11]. We also show that Maximum Pairs Orientation admits ratio $O(\log |P| / \log \log |P|)$, which is better than the ratio $O(\log n / \log \log n)$ of Gamzu et al. [WABI'10] when $|P|<n$.
\end{abstract}

\section{Introduction}

\subsection{Problems considered and our results}

We consider connectivity problems with orientation constraints. Unless stated otherwise, graphs are assumed to be undirected (and may not be simple), but we also consider directed graphs, and even mixed graphs, which may have both directed and undirected edges. Given a mixed graph $H$, an orientation of $H$

\footnotetext{
* A preliminary version of this article was presented at the 20th European Symposium on Algorithms (ESA 2012).

** Partially supported by National Science Centre grant no. N206 567140, Foundation for Polish Science, ERC Starting Grant NEWNET 279352, NSF CAREER award 1053605, DARPA/AFRL award FA8650-11-1-7162 and ONR YIP grant no. N000141110662.
}

$\star \star \star$ Partially supported by NSF support grant award number 0829959. 
is a directed graph $D$ obtained from $H$ by assigning to each undirected edge one of the two possible directions. For a mixed graph $H$ on node set $V$ and a multi-collection of ordered node pairs (that is convenient to consider as a set of directed edges) $P$ on $V$ let $P[H]$ denote the subset of the pairs (or edges) in $P$ for which $H$ contains a uv-path. We say that $H$ satisfies $P$ if $P[H]=P$, and that $H$ is $P$-orientable if $H$ admits an orientation $D$ that satisfies $P$. We note that for undirected graphs it is easy to check in polynomial time whether $H$ is $P$-orientable, cf. [10] and Section 3 in this paper. Let $n=|V|$ denote the number of nodes in $H$ and $m=|E(H)|+|P|$ the total number of edges and $\operatorname{arcs}$ in $H$ and ordered pairs in $P$.

Our first problem is the classic Steiner Forest problem with orientation constraints.

\author{
Steiner Forest Orientation \\ Instance: A graph $G=(V, E)$ with edge-costs and a set $P \subseteq V \times V$ of \\ ordered node pairs. \\ Objective: Find a minimum-cost subgraph $H$ of $G$ with an orientation $D$ that \\ satisfies $P$.
}

Theorem 1. Steiner Forest Orientation admits a 4-approximation algorithm.

Our next bunch of results deals with maximization problems of finding an orientation that satisfies the maximum number of pairs in $P$.

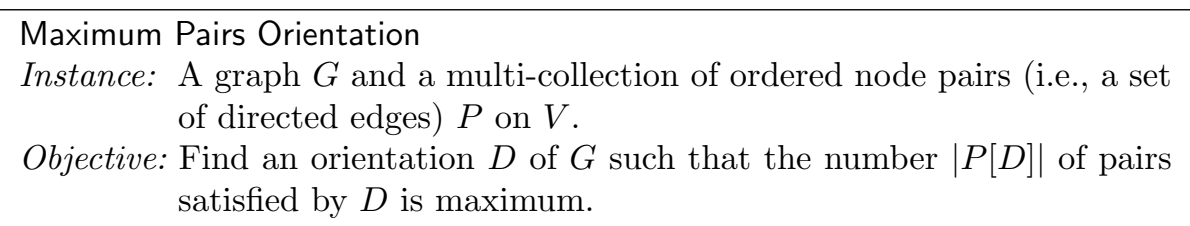

Let $k$ Pairs Orientation be the decision problem of determining whether Maximum Pairs Orientation has a solution of value at least $k$. Let $P$-Orientation be the decision problem of determining whether $G$ is $P$-orientable (this is the $k$ Pairs Orientation with $k=|P|)$. As was mentioned, for undirected graphs $P$ Orientation can be easily decided in polynomial time [10]. Arkin and Hassin [1] proved that on mixed graphs, $P$-Orientation is NP-complete, but it is polynomialtime solvable for $|P|=2$. Using new techniques, we widely generalize the result of [1] as follows.

Theorem 2. Given a mixed graph $G$ and $P \subseteq V \times V$ one can decide in $n^{O(|P|)}$ time whether $G$ is $P$-orientable; namely, $P$-Orientation with a mixed graph $G$ can be decided in $n^{O(|P|)}$ time. In particular, the problem can be decided in polynomial time for any instance with constant $|P|$.

In several papers, for example in [13], it is stated that any instance of Maximum Pairs Orientation admits a solution $D$ such that $|P[D]| \geq|P| /(4 \log n)$. Furthermore Gamzu et al. [7] show that Maximum Pairs Orientation admits an 
$O(\log n / \log \log n)$-approximation algorithm. In [3] it is shown that $k$ Pairs Orientation is fixed-parameter tractable ${ }^{4}$ when parameterized by the maximum number of pairs that can be connected via one node. They posed an open question if the problem is fixed-parameter tractable when parameterized by $k$ (the number of pairs that should be connected), namely, whether $k$ Pairs Orientation can be decided in $f(k)$ poly $(n)$ time, for some computable function $f$. By reducing a given instance and using already known results we answer this open question, moreover for $|P|<n$ we improve the approximation ratio $O(\log n / \log \log n)$ for Maximum Pairs Orientation of $[13,7]$.

Theorem 3. Any instance $(G, P)$ of Maximum Pairs Orientation, where $G$ is an undirected graph, admits a solution $D$, that can be computed in polynomial time, such that $|P[D]| \geq|P| /\left(4 \log _{2}(3|P|)\right)$. Furthermore

(i) $k$ Pairs Orientation can be decided in $O(n+m)+2^{O(k \cdot \log \log k)}$ time; thus it is fixed-parameter tractable when parameterized by $k$.

(ii) Maximum Pairs Orientation admits an $O(\log |P| / \log \log |P|)$-approximation algorithm.

Note that $|P|$ may be much smaller than $n$, say $|P|=2^{\sqrt{\log n}}$. While this size of $P$ does not allow exhaustive search in time polynomial in $n$, we do get an approximation ratio of $O(\sqrt{\log n} / \log \log n)$, which is better than the ratio $O(\log n / \log \log n)$ of Gamzu et al. [7].

Theorems 1, 2 and 3, are proved in sections 2, 3 and 4, respectively.

\subsection{Previous and related work}

Let Steiner Tree Orientation be a particular case of Steiner Forest Orientation when the pairs in $P$ form a star. Observe that one can reduce an instance of the classic Steiner Tree problem to an instance of Steiner Tree Orientation. Indeed, for an undirected graph $G=(V, E)$ and a set $T=\left\{t_{1}, \ldots, t_{k}\right\}$ of terminals, a subgraph $H$ of $G$ is connected and contains $T$ if and only if there is an orientation $D$ of $H$ satisfying $P=\left\{\left(t_{1}, t_{i}\right): 2 \leq i \leq k\right\}$. Since Steiner Tree is APX-hard [15], so is Steiner Tree Orientation.

One may also consider "high-connectivity" orientation problems, to satisfy prescribed connectivity demands. Let $\lambda_{H}(u, v)$ denote the $(u, v)$-edge-connectivity in a graph $H$, namely, the maximum number of pairwise edge-disjoint $u v$-paths in $H$. Similarly, let $\kappa_{H}(u, v)$ denote the $(u, v)$-node-connectivity in $H$, namely, the maximum number of pairwise internally node-disjoint $u v$-paths in $H$. Given an edge-connectivity demand function $r=\{r(u, v):(u, v) \in V \times V\}$, we say that $H$ satisfies $r$ if $\lambda_{H}(u, v) \geq r(u, v)$ for all $(u, v) \in V \times V$; similarly, for node

\footnotetext{
4 "Fixed-parameter tractable" means the following. In the parameterized complexity setting, an instance of a decision problem comes with an integer parameter $k$. A problem is said to be fixed-parameter tractable (w.r.t. $k$ ) if there exists an algorithm that decides any instance $(I, k)$ in time $f(k)$ poly $(|I|)$ for some (usually exponential) computable function $f$.
} 
connectivity demands, we say that $H$ satisfies $r$ if $\kappa_{H}(u, v) \geq r(u, v)$ for all $(u, v) \in V \times V$.

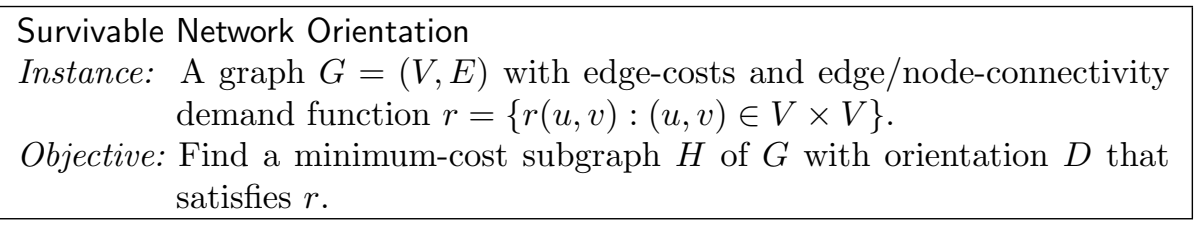

So far we assumed that the orienting costs are symmetric; this means that orienting an undirected edge connecting $u$ and $v$ in each one of the two directions is the same, namely, that $c(u, v)=c(v, u)$. This assumption is reasonable in practical problems, but in a theoretic more general setting, we might have nonsymmetric costs $c(u, v) \neq c(v, u)$. Note that the version with non-symmetric costs includes the min-cost version of the corresponding directed connectivity problem, and also the case when the input graph $G$ is a mixed graph, by assigning large/infinite costs to non-feasible orientations. For example, Steiner Forest Orientation with non-symmetric costs includes the Directed Steiner Forest problem, which is Label-Cover hard to approximate [2]. This is another reason to consider the symmetric costs version.

Khanna, Naor, and Shepherd [12] considered several orientation problems with non-symmetric costs. They showed that when $D$ is required to be $k$-edgeoutconnected from a given roots $s$ (namely, $D$ contains $k$ edge-disjoint paths from $s$ to every other node), then the problem admits a polynomial time algorithm. In fact they considered a more general problem of finding an orientation that covers an intersecting supermodular or crossing supermodular set-function. See [12] for precise definitions. Further generalization of this result due to Frank, T. Király, and Z. Király was presented in [6]. For the case when $D$ should be strongly connected, [12] obtained a 4-approximation algorithm; note that our Steiner Forest Orientation problem has much more general demands, that are not captured by intersecting supermodular or crossing supermodular setfunctions, but we consider symmetric edge-costs (otherwise the problem includes the Directed Steiner Forest problem). For the case when $D$ is required to be $k$ edge-connected, $k \geq 2,[12]$ obtained a pseudo-approximation algorithm that computes a $(k-1)$-edge-connected subgraph of cost at most $2 k$ times the cost of an optimal $k$-connected subgraph.

We refer the reader to [5] for a survey on characterization of graphs that admit orientations satisfying prescribed connectivity demands, and here mention only the following central theorem, that can be used to obtain a pseudo-approximation for edge-connectivity orientation problems.

Theorem 4 (Well-Balanced Orientation Theorem, Nash-Williams [14]). Any undirected graph $H=\left(V, E_{H}\right)$ has an orientation $D$ for which $\lambda_{D}(u, v) \geq$ $\left\lfloor\frac{1}{2} \lambda_{H}(u, v)\right\rfloor$ for all $(u, v) \in V \times V$.

We note that given $H$, an orientation as in Theorem 4 can be computed in polynomial time. It is easy to see that if $H$ has an orientation $D$ that satisfies $r$ 
then $H$ satisfies the demand function $q$ defined by $q(u, v)=r(u, v)+r(v, u)$. Theorem 4 implies that edge-connectivity Survivable Network Orientation admits a polynomial time algorithm that computes a subgraph $H$ of $G$ and an orientation $D$ of $H$ such that $c(H) \leq 2$ opt and

$\lambda_{D}(u, v) \geq\lfloor(r(u, v)+r(v, u)) / 2\rfloor \geq\lfloor\max \{r(u, v), r(v, u)\} / 2\rfloor \forall(u, v) \in V \times V$.

This is achieved by applying Jain's [11] algorithm to compute a 2-approximate solution $H$ for the corresponding undirected edge-connectivity Survivable Network instance with demands $q(u, v)=r(u, v)+r(v, u)$, and then computing an orientation $D$ of $H$ as in Theorem 4. This implies that if the costs are symmetric, then by cost at most 2opt we can satisfy almost half of the demand of every pair, and if also the demands are symmetric then we can satisfy all the demands. The above algorithm also applies for non-symmetric edge-costs, invoking an additional cost factor of $\max _{u v \in E} c(v, u) / c(u, v)$. Summarizing, we have the following observation, which we failed to find in the literature.

Corollary 5. Edge-connectivity Survivable Network Orientation (with non-symmetric costs) admits a polynomial time algorithm that computes a subgraph $H$ of $G$ and an orientation $D$ of $H$ such that $c(H) \leq 2$ opt $\cdot \max _{u v \in E} c(v, u) / c(u, v)$ and $\lambda_{D}(u, v) \geq\left\lfloor\frac{1}{2}(r(u, v)+r(v, u))\right\rfloor$ for all $(u, v) \in V \times V$. In particular, the problem admits a 2-approximation algorithm if both the costs and the demands are symmetric.

Not much is known about min-cost node-connectivity orientation problems. However, the following problem can be solved in polynomial time.

\section{$\ell$ Disjoint Paths Orientation}

Instance: A graph $G=(V, E)$ with edge-costs, $s, t \in V$, and an integer $\ell$.

Objective: Find a minimum-cost subgraph $H$ of $G$ with an orientation $D$ such that $D$ contains $\ell$ internally-disjoint $s t$-paths, and $\ell$ internallydisjoint $t s$-paths.

Checking whether $\ell$ Disjoint Paths Orientation admits a feasible solution can be done in polynomial time using the characterization of feasible solutions of Egawa, Kaneko, and Matsumoto, see Theorem 1 and Lemma 2 of [4].

Theorem 6 ([4]). Let $H=\left(V, E_{H}\right)$ be an undirected graph and let $s, t \in V$. Then $H$ has an orientation $D$ such that $\kappa_{D}(s, t), \kappa_{D}(t, s) \geq \ell$ if, and only if, $H$ contains $2 \ell$ edge-disjoint st-paths such that every $v \in V \backslash\{s, t\}$ belongs to at most 2 of them.

Therefore one can reduce $\ell$ Disjoint Paths Orientation to the Min-Cost $k$-Flow problem with node capacities, which is known to be solvable in polynomial time. Consequently, Theorem 6 implies the following.

Proposition 7. $\ell$ Disjoint Paths Orientation can be solved in polynomial time. 


\section{$2 \quad$ Algorithm for Steiner Forest Orientation (Theorem 1)}

In this section we prove Theorem 1. For a mixed graph or an edge set $H$ on a node set $V$ and $X, Y \subseteq V$ let $\delta_{H}(X, Y)$ denote the set of all (directed and undirected) edges in $H$ from $X$ to $Y$ and let $d_{H}(X, Y)=\left|\delta_{H}(X, Y)\right|$ denote their number; for brevity, $\delta_{H}(X)=\delta_{H}(X, \bar{X})$ and $d_{H}(X)=d_{H}(X, \bar{X})$, where $\bar{X}=V \backslash X$.

Given an integral set-function $f$ on subsets of $V$ we say that $H$ covers $f$ if $d_{H}(X) \geq f(X)$ for all $X \subseteq V$. Define a set-function $f_{r}$ by $f_{r}(\emptyset)=f_{r}(V)=0$ and for every $\emptyset \neq X \subset V$

$$
f_{r}(X)=\max \{r(u, v): u \in X, v \in \bar{X}\}+\max \{r(v, u): u \in X, v \in \bar{X}\} .
$$

Note that the set-function $f_{r}$ is symmetric, namely, that $f_{r}(X)=f_{r}(\bar{X})$ for all $X \subseteq V$.

Lemma 8. If an undirected edge set $H$ has an orientation $D$ that satisfies an edge-connectivity demand function $r$ then $H$ covers $f_{r}$.

Proof. Let $X \subseteq V$. By Menger's Theorem, any orientation $D$ of $H$ that satisfies $r$ has at least $\max \{r(u, v): u \in X, v \in \bar{X}\}$ edges from $X$ to $\bar{X}$, and at least $\max \{r(v, u): u \in X, v \in \bar{X}\}$ edges from $\bar{X}$ to $X$. The statement follows.

Recall that in the Steiner Forest Orientation problem we have $r(u, v)=1$ if $(u, v) \in P$ and $r(u, v)=0$ otherwise. We will show that if $r_{\max }=\max _{u, v \in V} r(u, v)=$ 1 then the inverse to Lemma 8 is also true, namely, if $H$ covers $f_{r}$ then $H$ has an orientation that satisfies $r$; for this case, we also give a 4-approximation algorithm for the problem of computing a minimum-cost subgraph that covers $f_{r}$. We do not know if these results can be extended for $r_{\max } \geq 2$.

Lemma 9. For $r_{\max }=1$, if an undirected edge set $H$ covers $f_{r}$ then $H$ has an orientation that satisfies $r$.

Proof. Observe that if $(u, v) \in P$ (namely, if $r(u, v)=1$ ) then $u, v$ belong to the same connected component of $H$. Hence it sufficient to consider the case when $H$ is connected. Let $D$ be an orientation of $H$ obtained as follows. Orient every 2edge-connected component of $H$ to be strongly connected (recall that a directed graph is strongly connected if there is a directed path from any of its nodes to any other); this is possible by Theorem 4 . Now we orient the bridges of $H$. Consider a bridge $e$ of $H$. The removal of $e$ partitions $V$ into two connected components $X, \bar{X}$. Note that $\delta_{P}(X, \bar{X})=\emptyset$ or $\delta_{P}(\bar{X}, X)=\emptyset$, since $f_{r}(X) \leq d_{H}(X)=1$. If $\delta_{P}(X, \bar{X}) \neq \emptyset$, we orient $e$ from $X$ to $\bar{X}$; if $\delta_{P}(\bar{X}, X) \neq \emptyset$, we orient $e$ from $\bar{X}$ to $X$; and if $\delta_{P}(X, \bar{X}), \delta_{P}(\bar{X}, X)=\emptyset$, we orient $e$ arbitrarily. It is easy to see that the obtained orientation $D$ of $H$ satisfies $P$.

We say that an edge-set or a graph $H$ covers a set-family $\mathcal{F}$ if $d_{H}(X) \geq 1$ for all $X \in \mathcal{F}$. A set-family $\mathcal{F}$ is said to be uncrossable if for any $X, Y \in \mathcal{F}$ the 
following holds: $X \cap Y, X \cup Y \in \mathcal{F}$ or $X \backslash Y, Y \backslash X \in \mathcal{F}$. The problem of finding a minimum-cost set of undirected edges that covers an uncrossable set-family $\mathcal{F}$ admits a primal-dual 2-approximation algorithm, provided the inclusion-minimal members of $\mathcal{F}$ can be computed in polynomial time [8]. It is known that the undirected Steiner Forest problem is a particular case of the problem of finding a min-cost cover of an uncrossable family, and thus admits a 2-approximation algorithm.

Lemma 10. Let $H=(V, J \cup P)$ be a mixed graph, where edges in $J$ are undirected and edges in $P$ are directed, such that for every $u v \in P$ both $u, v$ belong to the same connected component of the graph $(V, J)$. Then the set-family $\mathcal{F}=\left\{S \subseteq V: d_{J}(S)=1 \wedge d_{P}(S), d_{P}(\bar{S}) \geq 1\right\}$ is uncrossable, and its inclusion minimal members can be computed in polynomial time.

Proof. Let $\mathcal{C}$ be the set of connected components of the graph $(V, J)$. Let $C \in$ $\mathcal{C}$. Any bridge $e$ of $C$ partitions $C$ into two parts $C^{\prime}(e), C^{\prime \prime}(e)$ such that $e$ is the unique edge in $J$ connecting them. Note that the condition $d_{J}(S)=1$ is equivalent to the following condition $(\mathrm{C} 1)$, while if condition $(\mathrm{C} 1)$ holds then the condition $d_{P}(S), d_{P}(\bar{S}) \geq 1$ is equivalent to the following condition (C2) (since no edge in $P$ connects two distinct connected components of $(V, J)$ ).

(C1) There exists $C_{S} \in \mathcal{C}$ and a bridge $e_{S}$ of $C_{S}$, such that $S$ is a union of one of the sets $X^{\prime}=C_{S}^{\prime}\left(e_{S}\right), X^{\prime \prime}=C_{S}^{\prime \prime}\left(e_{S}\right)$ and sets in $\mathcal{C} \backslash\left\{C_{S}\right\}$.

(C2) $d_{P}\left(X^{\prime}, X^{\prime \prime}\right), d_{P}\left(X^{\prime \prime}, X^{\prime}\right) \geq 1$.

Hence we have the following characterization of the sets in $\mathcal{F}: S \in \mathcal{F}$ if, and only if, conditions (C1), (C2) hold for $S$. This implies that every inclusion-minimal member of $\mathcal{F}$ is $C^{\prime}(e)$ or $C^{\prime \prime}(e)$, for some bridge $e$ of $C \in \mathcal{C}$. In particular, the inclusion-minimal members of $\mathcal{F}$ can be computed in polynomial time.

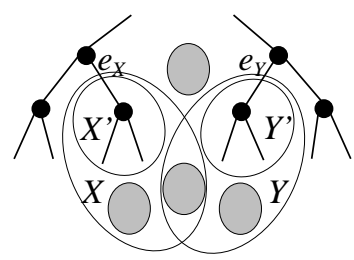

Fig. 1. Illustration to the proof of Lemma 10; components distinct from $C_{X}, C_{Y}$ are shown by gray ellipses.

Now let $X, Y \in \mathcal{F}$ (so conditions (C1), (C2) hold for each one of $X, Y$ ), let $C_{X}, C_{Y} \in \mathcal{C}$ be the corresponding connected components and $e_{X}, e_{Y}$ the corresponding bridges (possibly $C_{X}=C_{Y}$, in which case we also may have $e_{X}=e_{Y}$ ), and let $X^{\prime}, X^{\prime \prime}$ and $Y^{\prime}, Y^{\prime \prime}$ be the corresponding partitions of $C_{X}$ and $C_{Y}$, respectively. Since $e_{X}, e_{Y}$ are bridges, at least one of the sets $X^{\prime} \cap Y^{\prime}, X^{\prime} \cap$ $Y^{\prime \prime}, X^{\prime \prime} \cap Y^{\prime}, X^{\prime \prime} \cap Y^{\prime \prime}$ must be empty, say $X^{\prime} \cap Y^{\prime}=\emptyset$. Note that the set-family 
$\mathcal{F}$ is symmetric, hence to prove that $X \cap Y, X \cup Y \in \mathcal{F}$ or $X \backslash Y, Y \backslash X \in \mathcal{F}$, it is sufficient to prove that $A \backslash B, B \backslash A \in \mathcal{F}$ for some pair $A, B$ such that $A \in\{X, \bar{X}\}, B \in\{Y, \bar{Y}\}$. E.g., if $A=X$ and $B=\bar{Y}$, then $A \backslash B=X \cap Y$ and $B \backslash A=V \backslash(X \cup Y)$, hence $A \backslash B, B \backslash A \in \mathcal{F}$ together with the symmetry of $\mathcal{F}$ implies $X \cap Y, X \cup Y \in \mathcal{F}$. Similarly, if $A=\bar{X}$ and $B=\bar{Y}$, then $A \backslash B=Y \backslash X$ and $B \backslash A=X \backslash Y$, hence $A \backslash B, B \backslash A \in \mathcal{F}$ implies $Y \backslash X, X \backslash Y \in \mathcal{F}$. Thus w.l.o.g. we may assume that $X^{\prime} \subseteq X$ and $Y^{\prime} \subseteq Y$, see Figure 1, and we show that $X \backslash Y, Y \backslash X \in \mathcal{F}$. Recall that $X^{\prime} \cap Y^{\prime}=\emptyset$ and hence $X \cap Y$ is a (possibly empty) union of some sets in $\mathcal{C} \backslash\left\{C_{X}, C_{Y}\right\}$. Thus $X \backslash Y$ is a union of $X^{\prime}$ and some sets in $\mathcal{C} \backslash\left\{C_{X}, C_{Y}\right\}$. This implies that conditions (C1), (C2) hold for $X \backslash Y$, hence $X \backslash Y \in \mathcal{F}$; the proof that $Y \backslash X \in \mathcal{F}$ is similar. This concludes the proof of the lemma.

Lemma 11. Given a Steiner Forest Orientation instance, the problem of computing a minimum-cost subgraph $H$ of $G$ that covers $f_{r}$ admits a 4-approximation algorithm.

Proof. The algorithm has two phases. In the first phase we solve the corresponding undirected Steiner Forest instance with the same demand function $r$. The Steiner Forest problem admits a 2-approximation algorithm, hence $c(J) \leq 2$ opt. Let $J$ be a subgraph of $G$ computed by such a 2 -approximation algorithm. Note that $f_{r}(S)-d_{J}(S) \leq 1$ for all $S \subseteq V$. Hence to obtain a cover of $f_{r}$ it is sufficient to cover the family $\mathcal{F}=\left\{S \subseteq V: f_{r}(S)-d_{J}(S)=1\right\}$ of the deficient sets w.r.t. $J$. The key point is that the family $\mathcal{F}$ is uncrossable, and that the inclusion-minimal members of $\mathcal{F}$ can be computed in polynomial time. In the second phase we compute a 2-approximate cover of this $\mathcal{F}$ using the algorithm of [8]. Observe that the set $E(H) \backslash E(J)$, that is the set of edges of the optimum solution with edges of $J$ removed, covers the family $\mathcal{F}$ and therefore the cost of the second phase is at most 2opt. Consequently, the problem of covering $f_{r}$ is reduced to solving two problems of covering an uncrossable set-family.

To show that $\mathcal{F}$ is uncrossable we use Lemma 10. Note that for any $(u, v) \in P$ both $u, v$ belong to the same connected component of $(V, J)$, and that $f_{r}(S)-$ $d_{J}(S)=1$ if, and only if, $d_{J}(S)=1$ and $d_{P}(S), d_{P}(\bar{S}) \geq 1$, hence $\mathcal{F}=\{S \subseteq V$ : $\left.d_{J}(S)=1 \wedge d_{P}(S), d_{P}(\bar{S}) \geq 1\right\}$. Consequently, by Lemma 10 , the family $\mathcal{F}$ is uncrossable and its inclusion-minimal members can be computed in polynomial time. This concludes the proof of the lemma.

The proof of Theorem 1 is complete.

\section{Algorithm for $\boldsymbol{P}$-Orientation on mixed graphs (Theorem 2)}

In this section we prove Theorem 2. The following (essentially known) statement is straightforward.

Lemma 12. Let $G$ be a mixed graph, let $P$ be a set of directed edges on $V$, and let $C$ be a subgraph of $G$ that admits a strongly connected orientation. Let 
$G^{\prime}, P^{\prime}$ be obtained from $G, P$ by contracting $C$ into a single node. Then $G$ is $P$-orientable if, and only if, $G^{\prime}$ is $P^{\prime}$-orientable. In particular, this is so if $C$ is a cycle.

Corollary 13. P-orientation (with an undirected graph $G$ ) can be decided in polynomial time.

Proof. By repeatedly contracting a cycle of $G$, we obtain an equivalent instance, by Lemma 12 . Hence we may assume that $G$ is a tree. Then for every $(u, v) \in P$ there is a unique $u v$-path in $G$, which imposes an orientation on all the edges of this path. Hence if suffices to check that no two pairs in $P$ impose different orientations of the same edge of the tree.

Our algorithm for mixed graphs is based on a similar idea. We say that a mixed graph is an ori-cycle if it admits an orientation that is a directed simple cycle. We need the following statement, which was proved in [7] but we present the proof for completeness.

Lemma 14. Let $G=(V, E \cup A)$ be a mixed graph, where edges of $E$ are undirected and $A$ contains directed arcs, and let $G^{\prime}$ be obtained from $G$ by contracting every connected component of the undirected graph $(V, E)$ into a single node. If there is a directed cycle (possibly a self-loop) $C^{\prime}$ in $G^{\prime}$ then there is an ori-cycle $C$ in $G$, and such $C$ can be found in polynomial time.

Proof. If $C^{\prime}$ is also a directed cycle in $G$, then we take $C=C^{\prime}$. Otherwise, we replace every node $v_{X}$ of $C^{\prime}$ that corresponds to a contracted connected component $X$ of $(V, E)$ by a path, as follows. Let $a_{1}$ be the arc entering $v_{X}$ in $C^{\prime}$ and let $a_{2}$ be the arc leaving $v_{X}$ in $C^{\prime}$. Let $v_{1}$ be the head of $a_{1}$ and similarly let $v_{2}$ be a the tail of $a_{2}$. Since $X$ is a connected component in $(V, E)$, there is a $v_{1} v_{2}$-path in $(V, E)$, and we replace $X$ by this path. The result is the required ori-cycle $C$ (possibly a self-loop) in $G$. It is easy to see that such $C$ can be obtained from $C^{\prime}$ in polynomial time.

By Lemmas 14 and 12 we may assume that the directed graph $G^{\prime}$ obtained from $G$ by contracting every connected component of $(V, E)$, is a directed acyclic multigraph (with no self-loops). This preprocessing step is similar to the one used by Silverbush et al. [16]. Let $p=|P|$. Let $f: V \rightarrow V\left(G^{\prime}\right)$ be the function which for each node $v$ of $G$ assigns a node $f(v)$ in $G^{\prime}$ that represents the connected component of $(V, E)$ that contains $v$ (in other words the function $f$ shows a correspondence between nodes before and after contractions).

The first step of our algorithm is to guess the first and the last edge on the path for each of the $p$ pairs in $P$, by trying all $n^{O(p)}$ possibilities. If for the $i$-th pair an undirected edge is selected as the first or the last one on the corresponding path, then we orient it accordingly and move it from $E$ to $A$. Thus by functions last, first $:\{1, \ldots, p\} \rightarrow A$ we denote the guessed first and last arc for each of the $p$ paths.

Now we present a branching algorithm with exponential time complexity which we later convert to $n^{O(p)}$ time by applying a standard method of dynamic 
programming. Let $\pi$ be a topological ordering of $G^{\prime}$. By cur $:\{1, \ldots, p\} \rightarrow A$ we denote the most recently chosen arc from $A$ for each of the $p$ paths (initially $\operatorname{cur}(i)=\operatorname{first}(i))$. In what follows we consider subsequent nodes $v_{C}$ of $G^{\prime}$ with respect to $\pi$ and branch on possible orientations of the connected component $C$ of $G$. We use this orientation to update the function cur for all the arguments $i$ such that $\operatorname{cur}(i)$ is an arc entering a node mapped to $v_{C}$.

Let $v_{C} \in V\left(G^{\prime}\right)$ be the first node w.r.t. to $\pi$ which was not yet considered by the branching algorithm. Let $I \subseteq\{1, \ldots, p\}$ be the set of indices $i$ such that $\operatorname{cur}(i)=(u, v) \in A$ for $f(v)=v_{C}$, and $\operatorname{cur}(i) \neq \operatorname{last}(i)$. If $I=\emptyset$ then we skip $v_{C}$ and proceed to the next node in $\pi$. Otherwise for each $i \in I$ we branch on choosing an $\operatorname{arc}(u, v) \in A$ such that $f(u)=v_{C}$, that is we select an arc that the $i$-path will use just after leaving the connected component of $G$ corresponding to the node $v_{C}$ (note that there are at most $|A|^{|I|}=n^{O(p)}$ branches). Before updating the $\operatorname{arcs} \operatorname{cur}(i)$ for each $i \in I$ in a branch, we check whether the connected component $C$ of $(V, E)$ consisting of nodes $f^{-1}\left(v_{C}\right)$ is accordingly orientable by using Corollary 13 (see Figure 2). Finally after considering all the nodes in $\pi$ we check whether for each $i \in\{1, \ldots, p\}$ we have $\operatorname{cur}(i)=\operatorname{last}(i)$. If this is the case our algorithm returns YES and otherwise it returns NO in this branch.
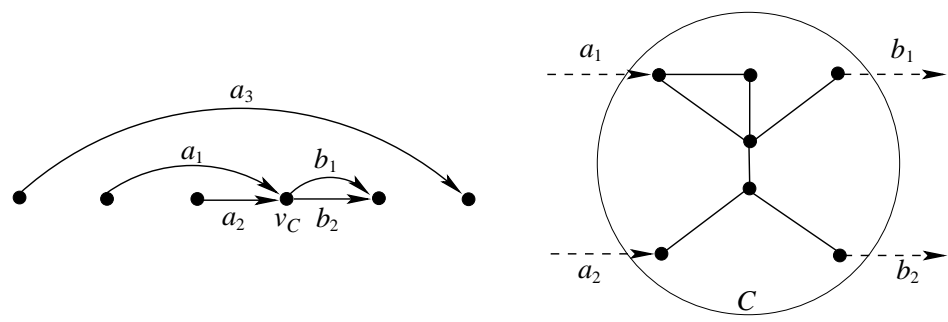

Fig. 2. Our algorithm considers what orientation the connected component $C$ (of $(V, E))$ will have. Currently we have $\operatorname{cur}(1)=a_{1}, \operatorname{cur}(2)=a_{2}$ and $\operatorname{cur}(3)=a_{3}$, hence $I=\{1,2\}$. If in a branch we set new values $\operatorname{cur}(1)=b_{1}$ and $\operatorname{cur}(2)=b_{2}$ then by Corollary 13 we can verify that it is possible to orient $C$, so that there is a path from the end-point of $a_{1}$ to the start-point of $b_{1}$ and from the end-point of $a_{2}$ to the startpoint of $b_{2}$. However the branch with new values $\operatorname{cur}(1)=b_{2}$ and $\operatorname{cur}(2)=b_{1}$ will be terminated, since it is not possible to orient $C$ accordingly.

The correctness of our algorithm follows from the invariant that each node $v_{C}$ is considered at most once, since all the updated values $\operatorname{cur}(i)$ are changed to arcs that are to the right with respect to $\pi$.

Observe that when considering a node $v_{C}$ it is not important what orientations previous nodes in $\pi$ have, because all the relevant information is contained in the cur function. Therefore to improve the currently exponential time complexity we apply the standard technique of dynamic programming (memoization), that is, store results of all the previously computed recursive calls. Consequently 
for any index of the currently considered node in $\pi$ and any values of the function cur, there is at most one branch for which we compute the result, since for the subsequent recursive calls we use the previously computed results. This leads to $n^{O(p)}$ branches and $n^{O(p)}$ total time and space complexity.

\section{Algorithms for Maximum Pairs Orientation (Theorem 3)}

In this section we prove Theorem 3.

Lemma 15. There exists a linear time algorithm that given an instance of Maximum Pairs Orientation or of $\mathrm{k}$ Pairs Orientation, transforms it into an equivalent instance such that the input graph is a tree with at most $3 p-1$ nodes.

Proof. As is observed in [10], and also follows from Lemma 12, we can assume that the input graph $G$ is a tree; such a tree can be constructed in linear time by contracting the 2-edge-connected components of $G$. For each edge $e$ of $G$ we compute an integer $P(e)$, that is the number of pairs $(s, t)$ in $P$, such that $e$ belongs to the shortest path between $s$ and $t$ in $G$. If for an edge $e$ we have $P(e) \leq 1$, we contract this edge, since we can always orient it as desired by at most one $s t$-path. Note that after this operation each leaf belongs to at least two pairs in $P$. If a node $v$ has degree 2 in the tree and does not belong to any pair, we contract one of the edges incident to $v$; this is since in any inclusion minimal solution, one of the two edges enters $v$ if, and only if, the other leaves $v$.

The linear time implementation of the presented reductions is as follows. First, using a linear time algorithm for computing 2-edge-connected components, and by scanning every edge in $E \cup P$, we can see which components it connects, thus obtaining an equivalent instance where $G$ is a tree. To compute all the values $P(e)$, we root the tree in an arbitrary node and create a multiset of pairs $P^{\prime}=\{(s, \operatorname{lca}(s, t)),(t, \operatorname{lca}(s, t)):(s, t) \in P\}$, where lca $(s, t)$ is the lowest common ancestor of $s$ and $t$, which can be computed in linear time [9]. Note that in $P^{\prime}$ the first coordinate of each pair is an descendant of the second coordinate node and pairs in $P^{\prime}$ represent upward paths. Let $a(v)=\left|\left\{(v, x) \in P^{\prime}\right\}\right|-\left|\left\{(x, v) \in P^{\prime}\right\}\right|$ and observe that if $u$ is a parent of $v$ in the tree $G$, then $P(u v)$ equals the sum of values $a\left(u^{\prime}\right)$ over all descendants $u^{\prime}$ of the node $u$ (including $u^{\prime}=u$ ). Therefore we can compute all the values $P(e)$ in linear time and contract all the edges with $P(e)=1$. Note that after the contractions are done we need to relabel pairs in $P$, since some nodes may have their labels changed, but this can be also done in linear time by storing a new label for each node in a table. Finally using a graph search algorithm we find maximal paths such that each internal node is of degree two and does not belong to any pair. For each such path we contract all but one edge.

We claim that after these reductions are implemented, the tree $G^{\prime}$ obtained has at most $3 p-1$ nodes. Let $\ell$ be the number of leaves and $t$ the number of nodes of degree 2 in $G^{\prime}$. As each node of degree less than 3 in $G^{\prime}$ is an $s_{i}$ or $t_{i}$, $\ell+t \leq 2 p$. Since each leaf belongs to at least two pairs in $P, \ell \leq p$. The number of nodes of degree at least 3 is at most $\ell-1$ and so $\left|V\left(G^{\prime}\right)\right| \leq 2 \ell+t-1 \leq 3 p-1$. This concludes the proof of the lemma. 
After applying Lemma 15, the number of nodes $n$ of the returned tree is at most $3 p-1$. Therefore, by [13], one can find in polynomial time a solution $D$, such that $|P[D]| \geq p /\left(4 \log _{2} n\right) \geq p /\left(4 \log _{2}(3 p)\right)$. Therefore, if for a given $k$ Pairs Orientation instance we have $k \leq p /\left(4 \log _{2}(3 p)\right)$, then clearly it is a YES instance. However if $k>p /\left(4 \log _{2}(3 p)\right)$, then $p=\Theta(k \log k)$. In order to solve the $k$ Pairs Orientation instance we consider all possible $\left(\begin{array}{l}p \\ k\end{array}\right)$ subsets $P^{\prime}$ of exactly $k$ pairs from $P$, and check if the graph is $P^{\prime}$-orientable. Observe that

$$
\left(\begin{array}{l}
p \\
k
\end{array}\right) \leq \frac{p^{k}}{k !} \leq \frac{p^{k}}{(k / e)^{k}} \leq \frac{p^{k}}{\left(p /\left(4 e \log _{2}(3 p)\right)\right)^{k}}=\left(4 e \log _{2}(3 p)\right)^{k}=2^{O(k \log \log k)}
$$

where the second inequality follows from Stirling's formula. Therefore the running time is $O(m+n)+2^{O(k \log \log k)}$, which proves (i).

Combining Lemma 15 with the $O(\log n / \log \log n)$-approximation algorithm of Gamzu et al. [7] proves (ii). Thus the proof of Theorem 3 is complete.

\section{Conclusions and open problems}

In this paper we considered minimum-cost and maximum pairs orientation problems. Our main results are a 4-approximation algorithm for Steiner Forest Orientation, an $n^{O(|P|)}$ time algorithm for P-Orientation on mixed graphs, and an $O(n+m)+2^{O(k \cdot \log \log k)}$ time algorithm for $k$ Pairs Orientation (which implies that $k$ Pairs Orientation is fixed-parameter tractable when parameterized by $k$, solving an open question from [3]). We now mention some open problems, most of them related to the work of Khanna, Naor, and Shepherd [12].

We have shown that the $P$-Orientation problem on mixed graphs parameterized by $|P|$ belongs to XP, however to the best of our knowledge it is not known whether this problem is fixed parameter tractable or $W[1]$-hard.

As was mentioned, [12] showed that the problem of computing a minimumcost $k$-edge-outconnected orientation can be solved in polynomial time, even for non-symmetric edge-costs. To the best of our knowledge, for node-connectivity, and even for the simpler notion of element-connectivity, no non-trivial approximation ratio is known even for symmetric costs and $k=2$. Moreover, even the decision version of determining whether an undirected graph admits a 2outconnected orientation is not known to be in $\mathrm{P}$ nor NP-complete.

For the case when the orientation $D$ is required to be $k$-edge-connected, $k \geq 2,[12]$ obtained a pseudo-approximation algorithm that computes a $(k-1)$ edge-connected subgraph of cost at most $2 k$ times the cost of an optimal $k$ connected subgraph. It is an open question if the problem admits a non-trivial true approximation algorithm even for $k=2$.

\section{Acknowledgements}

We are thankful to anonymous referees for their helpful comments and remarks. 


\section{References}

1. E. Arkin and R. Hassin. A note on orientations of mixed graphs. Discrete Applied Mathematics, 116(3):271-278, 2002.

2. Y. Dodis and S. Khanna. Design networks with bounded pairwise distance. In STOC, pages 750-759, 1999.

3. B. Dorn, F. Hüffner, D. Krüger, R. Niedermeier, and J. Uhlmann. Exploiting bounded signal flow for graph orientation based on cause-effect pairs. Algorithms for Molecular Biology, 6(21), 2011.

4. Y. Egawa, A. Kaneko, and M. Matsumoto. A mixed version of Menger's theorem. Combinatorica, 11:71-74, 1991.

5. A. Frank and T. Király. Combined connectivity augmentation and orientation problems. Discrete Applied Mathematics, 131(2):401-419, 2003.

6. A. Frank, T. Király, and Z. Király. On the orientation of graphs and hypergraphs. Discrete Applied Mathematics, 131:385400, 2003.

7. I. Gamzu, D. Segev, and R. Sharan. Improved orientations of physical networks. In $W A B I$, pages 215-225, 2010.

8. M. Goemans, A. Goldberg, S. Plotkin, D. Shmoys, E. Tardos, and D. Williamson. Improved approximation algorithms for network design problems. In SODA, pages 223-232, 1994

9. D. Harel and R. E. Tarjan. Fast algorithms for finding nearest common ancestors. SIAM J. Comput., 13(2):338-355, 1984.

10. R. Hassin and N. Megiddo. On orientations and shortest paths. Linear Algebra and Its Applications, pages 589-602, 1989.

11. K. Jain. A factor 2 approximation algorithm for the generalized Steiner network problem. Combinatorica, 21(1):39-60, 2001.

12. S. Khanna, J. Naor, and B. Shepherd. Directed network design with orientation constraints. In SODA, pages 663-671, 2000.

13. A. Medvedovsky, V. Bafna, U. Zwick, and R. Sharan. An algorithm for orienting graphs based on cause-effect pairs and its applications to orienting protein networks. In WABI, pages 222-232, 2008.

14. Nash-Williams. On orientations, connectivity and odd vertex pairings in finite graphs. Canad. J. Math., 12:555-567, 1960.

15. L. K. Platzman and I. John J. Bartholdi. Spacefilling curves and the planar traveling salesman problem. Journal of the ACM, 36(4):719-737, 1989.

16. D. Silverbush, M. Elberfeld, and R. Sharan. Optimally orienting physical networks. In $R E C O M B$, pages 424-436, 2011. 